Pacific Journal of Mathematic 


\title{
PRIMARY ABELIAN GROUPS MODULO FINITE GROUPS
}

\author{
RONALD J. ENSEY
}

\begin{abstract}
Let $\mathscr{A}$ be the category of Abelian groups, $\mathscr{F}$ the Serre class of finite Abelian groups, and form the quotient category $\mathscr{A} / \mathscr{F}$. The purpose of this paper is to find a complete set of invariants for direct sums of countable reduced p-groups, such groups being considered as objects of the category $\mathscr{A} / \mathscr{F}$. Specifically, it will be shown that two direct sums of countable reduced p-groups $G$ and $H$ are isomorphic in $\mathscr{A} / \mathscr{F}$ if and only if and

$f_{G}(\alpha)=f_{H}(\alpha)$ for almost all ordinal numbers $\alpha$,

$f_{G}(\alpha) \neq f_{H}(\alpha)$ implies $\max \left(f_{G}(\alpha), f_{H}(\alpha)\right)<f_{0}$.
\end{abstract}

The objects of the quotient category $\mathscr{A} / \mathscr{L}$ where $\mathscr{L}$ is any Serre class of $\mathscr{A}$ are just the objects of $\mathscr{A}$. A description of Hom $\mathscr{A} / \mathscr{L}(G, H)$ for objects $G$ and $H$ of $\mathscr{A} / \mathscr{L}$ can be found in [5]. Such a description is omitted here since two Abelian groups $G$ and $H$ are isomorphic in $\mathscr{A} / \mathscr{L}$ if and only if there exist subgroups $S$ and $A$ of $G$, and $T$ and $B$ of $H$ such that $S / A \simeq T / B$ in $\mathscr{A}$ and $G / S, A, H / T, B \in \mathscr{L}$. (See 2.3 and 2.4 in [5].) Consequently all computations can be made in $\mathscr{A}$ the category of Abelian groups. In particular the words group and homomorphism will always mean Abelian group and $\mathscr{A}$-homomorphism, respectively. For a proof that $\mathscr{A} / \mathscr{L}$ is an Abelian category, see [3].

The following is immediate.

Proposition 1. If two groups $G$ and $H$ are isomorphic in the category $\mathscr{A} \mid \mathscr{F}$, then either $|G|=|H|$ or $\max (|G|,|H|)<\aleph_{0}$.

Let $G$ be a reduced $p$-group, and let $\alpha$ be an ordinal number. Define $p^{\alpha} G$ inductively as follows: $p^{0} G=G, p^{\alpha} G=p\left(p^{\alpha-1} G\right)$ if $\alpha-1$ exists, and $p^{\alpha} G=\bigcap_{\beta<\alpha} p^{\beta} G$ if $\alpha$ is a limit ordinal. The dimension $f_{G}(\alpha)$ of the vector space $\left(p^{\alpha} G\right)[p] /\left(p^{\alpha+1} G\right)[p]$ is called the $\alpha$ th Ulm invariant of $G$. Define $G^{\alpha}$ inductively as follows: $G^{0}=G, G^{\alpha}=p^{\omega}\left(G^{\alpha-1}\right)$ if $\alpha-1$ exists, and $G^{\alpha}=\bigcap_{\beta<\alpha} G^{\beta}$ if $\alpha$ is a limit ordinal. Since $G$ is reduced, there is a least ordinal $\tau,|\tau| \leqq|G|$, such that $G^{\tau}=0$. The quotient groups $G^{\alpha} / G^{\alpha+1}, \alpha<\tau$, are called the Ulm factors of $G$. All the Ulm factors except possibly the last, if it exists, are unbounded. An element $x \in G$ has height $\alpha$ in $G$ if $x \in p^{\alpha} G$, but $x \notin p^{\alpha+1} G$. Ulm's theorem states that two countable reduced $p$-groups are isomorphic if 
and only if their corresponding Ulm factors are isomorphic, or equivalently, if and only if they have the same Ulm invariants. This result has been extended to direct sums of countable reduced $p$-groups by Kolettis in [4]. The author has shown in [1] that two direct sums of countable reduced $p$-groups $G$ and $H$ are isomorphic in the quotient category $\mathscr{A} \mid \mathscr{B}$, where $\mathscr{B}$ is the Serre class of bounded groups, if and only if there is an integer $k \geqq 0$ such that for all ordinal numbers $\alpha$ and integers $r \geqq 0$

$$
\sum_{j=0}^{r} f_{G}(\alpha+k+j) \leqq \sum_{j=0}^{r+2 k} f_{H}(\alpha+j)
$$

and

$$
\sum_{j=0}^{r} f_{H}(\alpha+k+j) \leqq \sum_{j=0}^{r+2 k} f_{G}(\alpha+j) .
$$

Since the Serre class of finite groups is a subclass of the Serre class of bounded groups, it is natural to ask how the above conditions must be strengthened to characterize isomorphism in $\mathscr{A} / \mathscr{F}$. That is the intention of this paper. Any unexplained notation or terminology will be that of Fuchs in [2] with the exception that $G+H$ will denote the sum of $G$ and $H$ while $G \oplus H$ will be the direct sum.

Lemma 2. Let $S$ be a cofinite subgroup of a reduced p-group $G$. Then there is an integer $n \geqq 0$ such that $f_{G}(\alpha)=f_{S}(\alpha)$ for all ordinal numbers $\alpha \geqq n$.

Proof. Since $S$ is cofinite in $G$, write $G / S=\left\{x_{1}+S, \cdots, x_{r}+S\right\}$. Let $n \geqq 0$ be an integer such that $p^{n} x_{i}=0$ for $i=1, \cdots, r$. Let $p^{n} y \in p^{n} G$. Then $y+S=x_{i}+S$ for some $i$ among $1, \cdots, r$. Thus $y-x_{i} \in S$ and $p^{n} y=p^{n}\left(y-x_{i}\right) \in p^{n} S$. Hence $p^{n} G=p^{n} S$ and $f_{G}(\alpha)=$ $f_{S}(\alpha)$ for all ordinal numbers $\alpha \geqq n$.

LEMma 3. Let $S$ be a cofinite subgroup of a reduced p-group $G$. Then for any integer $n \geqq 0, f_{G}(n)=f_{S}(n)$ or $\max \left(f_{G}(n), f_{S}(n)\right)<\boldsymbol{\aleph}_{0}$.

Proof. Let $f:\left(p^{n} S\right)[p] /\left(p^{n+1} S\right)[p] \rightarrow\left(p^{n} G\right)[p] /\left(p^{n+1} G\right)[p]$ be the homomorphism induced by the natural injection $S \rightarrow G$. Then Ker $f=$ $\left(\left(p^{n} S\right)[p] \cap\left(p^{n+1} G\right)[p]\right) /\left(p^{n+1} S\right)[p]$ and $|\operatorname{Ker} f| \leqq\left|\left(p^{n+1} G\right)[p] /\left(p^{n+1} S\right)[p]\right| \leqq$ $\left|\left(p^{n+1} G / p^{n+1} S\right)[p]\right| \leqq\left|p^{n+1} G / p^{n+1} S\right| \leqq|G / S| . \quad$ Also $\quad \operatorname{Im} f=\left(\left(p^{n} S\right)[p]+\right.$ $\left.\left(p^{n+1} G\right)[p]\right) /\left(p^{n+1} G\right)[p]$ and $|\operatorname{Coker} f|=\left|\left(p^{n} G\right)[p] /\left(\left(p^{n} S\right)[p]+\left(p^{n+1} G\right)[p]\right)\right| \leqq$ $\left|\left(p^{n} G\right)[p] /\left(p^{n} S\right)[p]\right| \leqq|G / S|$. Thus $\left(p^{n} S\right)[p] /\left(p^{n+1} S\right)[p]$ and $\left(p^{n} G\right)[p] /$ $\left(p^{n+1} G\right)[p]$ are isomorphic in $\mathscr{A} / \mathscr{F}$. The lemma now follows from Proposition 1.

LEMma 4. Let $\beta$ be an ordinal number, and let $A$ be a subgroup 
of a reduced p-group $G$ such that $A$ contains no elements of height $\beta$ or $\beta+1$, the heights taken in $G$. Then $f_{G / A}(\beta)=f_{G}(\beta)$. In particular if $A$ is a finite subgroup of $G$, then $f_{G / A}(\alpha)=f_{G}(\alpha)$ for almost all ordinal numbers $\alpha$.

Proof. Let $\left\{x_{i}+A+\left(\left(p^{\beta+1} G+A\right) / A\right)[p]\right\}_{I}$ be a basis of

$$
\left(\left(p^{\beta} G+A\right) / A\right)[p] /\left(\left(p^{\beta+1} G+A\right) / A\right)[p]
$$

with $x_{i} \in p^{\beta} G$ for each $i \in I$. Then $p x_{i} \in p^{\beta+1} G \cap A$, and since $A$ has no elements of height $\beta+1$ in $G, p x_{i} \in p^{\beta+2} G$. Write $p x_{i}=p z_{i}$ where $z_{i} \in p^{\beta+1} G$. Then $x_{i}-z_{i} \in\left(p^{\beta} G\right)[p]$, but $x_{i}-z_{i} \notin\left(p^{\beta+1} G\right)[p]$. Also $x_{i}-$ $z_{i}+A+\left(\left(p^{\beta+1} G+A\right) / A\right)[p]=x_{i}+A+\left(\left(p^{\beta+1} G+A\right) / A\right)[p]$. Let $y_{i}=$ $x_{i}-z_{i}$ for $i \in I$. Then $\left\{y_{i}+A+\left(\left(p^{\beta+1} G+A\right) / A\right)[p]\right\}_{I}$ is a basis of

$$
\left(\left(p^{\beta} G+A\right) / A\right)[p] /\left(\left(p^{\beta+1} G+A\right) / A\right)[p]
$$

with $y_{i} \in\left(p^{\beta} G\right)[p]$ for each $i \in I$. Suppose $\sum_{i \in J} r_{i} y_{i} \in\left(p^{\beta+1} G\right)[p]$ for some finite subset $J \subseteq I$. Then $\sum_{i \in J} r_{i} y_{i}+A \in\left(\left(p^{\beta+1} G+A\right) / A\right)[p]$. Thus $p \mid r_{i}$ for each $i \in J$ and $\left\{y_{i}+\left(p^{\beta+1} G\right)[p]\right\}_{I}$ is an independent subset of $\left(p^{\beta} G\right)[p] /\left(p^{\beta+1} G\right)[p]$. Hence $f_{G / A}(\beta) \leqq f_{G}(\beta)$.

Let $\left\{x_{i}+\left(p^{\beta+1} G\right)[p]\right\}_{K}$ be a basis of $\left(p^{\beta} G\right)[p] /\left(p^{\beta+1} G\right)[p]$. Suppose $\sum_{i \in L} s_{i} x_{i}+A \in\left(\left(p^{\beta+1} G+A\right) / A\right)[p]$ for some finite subset $L \subseteq K$. Then $\sum_{i \in L} s_{i} x_{i}+A=p x+A$ for some $x \in p^{\beta} G$. Now $y=\sum_{i \in L} s_{i} x_{i}$ $p x \in p^{\beta} G \cap A$. Since $A$ has no elements of height $\beta$ in $G, y \in p^{\beta+1} G$. So

$$
\sum_{i \in L} s_{i} x_{i} \in\left(p^{\beta+1} G\right)[p],
$$

and $p \mid s_{i}$ for each $i \in L$. Thus $\left\{x_{i}+A+\left(\left(p^{\beta+1} G+A\right) / A\right)[p]\right\}_{K}$ is an independent subset of $\left(\left(p^{\beta} G+A\right) / A\right)[p] /\left(\left(p^{\beta+1} G+A\right) / A\right)[p]$. Hence $f_{G}(\beta) \leqq f_{G / A}(\beta)$.

Lemma 5. Let $G$ be a reduced p-group with finite subgroup $A$. Then for any ordinal number $\alpha, f_{G}(\alpha)=f_{G / A}(\alpha)$ or $\max \left(f_{G}(\alpha)\right.$, $\left.f_{G / A}(\alpha)\right)<\boldsymbol{\aleph}_{0}$.

Proof. The projection $G \rightarrow G / A$ induces a homomorphism

$$
f:\left(p^{\alpha} G\right)[p] /\left(p^{\alpha+1} G\right)[p] \longrightarrow\left(\left(p^{\alpha} G+A\right) / A\right)[p] /\left(\left(p^{\alpha+1} G+A\right) / A\right)[p] .
$$

The kernel of $f$ is $\left(\left(p^{\alpha} G\right)[p] \cap\left(p^{\alpha+1} G+A\right)[p]\right) /\left(p^{\alpha+1} G\right)[p]$ and therefore

$$
\begin{aligned}
|\operatorname{Ker} f| & \leqq \mid\left(p^{\alpha+1} G+A\right)[p] /\left(p^{\alpha+1} G[p]|\leqq|\left(\left(p^{\alpha+1} G+A\right) / p^{\alpha+1} G\right)[p] \mid\right. \\
& \leqq\left|\left(p^{\alpha+1} G+A\right) / p^{\alpha+1} G\right|=\left|A / p^{\alpha+1} G \cap A\right| \leqq|A| .
\end{aligned}
$$

Also $\operatorname{Im} f=\left(\left(\left(p^{\alpha} G\right)[p]+p^{\alpha+1} G+A\right) / A\right)[p] /\left(\left(p^{\alpha+1} G+A\right) / A\right)[p]$, Coker $f$ 
$\simeq\left(\left(p^{\alpha} G+A\right) / A\right)[p] /\left(\left(\left(p^{\alpha} G\right)[p]+p^{\alpha+1} G+A\right) / A\right)[p], \quad$ and $\quad \mid$ Coker $f \mid \leqq$ $\left|\left(\left(p^{\alpha} G+A\right) / A\right)[p] /\left(\left(\left(p^{\alpha} G\right)[p]+A\right) / A\right)[p]\right|$. Let $x+A, y+A \in\left(\left(p^{\alpha} G+\right.\right.$ $A) / A)[p]$ with $x, y \in p^{\alpha} G$. Then $p x, p y \in A$. If $p x=p y$, then $x-$ $y \in\left(p^{\alpha} G\right)[p], x-y+A \in\left(\left(\left(p^{\alpha} G\right)[p]+A\right) / A\right)[p]$, and $x+A+\left(\left(\left(p^{\alpha} G\right)[p]+\right.\right.$ $A) / A)[p]=y+A+\left(\left(\left(p^{\alpha} G\right)[p]+A\right) / A\right)[p]$. So $\quad \mid$ Coker $f|\leqq|\left(\left(p^{\alpha} G+\right.\right.$ $A) / A)[p] /\left(\left(\left(p^{\alpha} G\right)[p]+A\right) / A\right)[p]|\leqq| A \mid$. Hence $\left(p^{\alpha} G\right)[p] /\left(p^{\alpha+1} G\right)[p]$ and $\left(\left(p^{\alpha} G+A\right) / A\right)[p] /\left(\left(p^{\alpha+1} G+A\right) / A\right)[p]$ are isomorphic in $\mathscr{A} / \mathscr{F}$. The lemma now follows from Proposition 1.

Lemma 6. Let $\beta$ be an ordinal number, and let $G$ and $H$ be countable reduced p-groups such that

(i) $f_{G}(\alpha)=f_{H}(\alpha)$ for all ordinal number $\alpha \neq \beta$, and

(ii) $\max \left(f_{G}(\beta), f_{H}(\beta)\right)<\boldsymbol{\aleph}_{0}$.

Then $G$ and $H$ are isomorphic in $\mathscr{A} / \mathscr{F}$.

Proof. Take $f_{G}(\beta) \geqq f_{H}(\beta)$, and let $k \geqq 0$ be an integer such that $f_{G}(\beta)=f_{H}(\beta)+k$. Write $\beta=\omega \gamma+n, n \geqq 0$. Then $G_{\gamma} \simeq H_{r} \oplus$ $\sum_{k} C\left(p^{n+1}\right)$ where $G_{\gamma}=G^{\gamma} / G^{\gamma+1}$ and $H_{\gamma}=H^{\gamma} / H^{\gamma+1}$. Suppose $H_{\gamma}$ is finite. Then so is $G_{\gamma}$. Moreover, $G / G^{\gamma} \simeq H / H^{\gamma}$, and so $G$ and $H$ are isomorphic in $\mathscr{A} / \mathscr{F}$. Assume $H_{\gamma}$ is infinite. For each $\alpha<\gamma$, write $H_{\alpha}=$ $L_{\alpha} \oplus M_{\alpha}$ where $L_{\alpha}$ and $M_{\alpha}$ are unbounded and $\left|L_{\alpha}\right|=\left|M_{\alpha}\right|=\left|H_{\alpha}\right|$. Let $L$ be a countable reduced $p$-group whose Ulm factors are $H_{\alpha}$ for $\alpha \geqq \gamma$ and $L_{\alpha}$ for $\alpha<\gamma$. (Such a group exists by Zippin's lemma.) Let $M$ be a countable reduced $p$-group whose Ulm factors are $M_{\alpha}$ for $\alpha<\gamma$ and $M_{\gamma}=\sum_{k} C\left(p^{n+1}\right)$. Then $L \oplus M \simeq G$ and $L \oplus\left(M / M^{r}\right) \simeq H$. Hence $G$ and $H$ are isomorphic in $\mathscr{A} / \mathscr{F}$.

Lemma 7. Let $\beta$ be an ordinal number, and let $G$ and $H$ be direct sums of countable reduced p-groups such that

(i) $f_{G}(\alpha)=f_{H}(\alpha)$ for all ordinal numbers $\alpha \neq \beta$, and

(ii) $\max \left(f_{G}(\beta), f_{H}(\beta)\right)<\boldsymbol{\aleph}_{0}$.

Then $G$ and $H$ are isomorphic in $\mathscr{A} / \mathscr{F}$.

Proof. Let $G=\sum_{\lambda \in \Lambda}^{\prime} X_{\lambda}$ where $\left|X_{\lambda}\right| \leqq \boldsymbol{\aleph}_{0}$ for each $\lambda \in \Lambda$. Let $\Lambda_{1}=\left\{\lambda \in \Lambda \mid f_{X_{\lambda}}(\beta)=0\right\}$, and let $X=\sum_{\lambda \in \Lambda-\Lambda_{1}} X_{\lambda}$. Since $f_{G}(\beta)$ is finite, $\left|A-A_{1}\right|<\boldsymbol{\aleph}_{0}$. There is a countable reduced $p$-group $Y$ with $f_{Y}(\alpha)=$ $f_{X}(\alpha)$ for $\alpha \neq \beta$ and $f_{Y}(\beta)=f_{H}(\beta)$. By Lemma $6, X$ and $Y$ are isomorphic in $\mathscr{A} / \mathscr{F}$. Hence $\sum_{\lambda \in \lambda_{1}} X_{\lambda} \oplus X$ and $\sum_{\lambda \in A_{1}} X_{\lambda} \oplus Y$ are isomorphic in $\mathscr{A} / \mathscr{F}$. But $G \simeq \sum_{\lambda \in \Lambda_{1}} X_{\lambda} \oplus X$ and $H \simeq \sum_{\lambda \in \Lambda_{1}} X_{\lambda} \oplus Y$. Hence $G$ and $H$ are isomorphic in $\mathscr{A} / \mathscr{F}$.

Theorem. Let $G$ and $H$ be direct sums of countable reduced $p$ groups. Then $G$ and $H$ are isomorphic in $\mathscr{A} / \mathscr{F}$ if and only if 

and

(i) $f_{G}(\alpha)=f_{H}(\alpha)$ for almost all ordinal numbers $\alpha$,

(ii) $f_{G}(\alpha) \neq f_{H}(\alpha)$ implies $\max \left(f_{G}(\alpha), f_{H}(\alpha)\right)<\boldsymbol{\aleph}_{0}$.

Proof. That (i) and (ii) are necessary follows from Lemmas 2, 3, 4, and 5. That (i) and (ii) are sufficient follows from a finite number of applications of Lemma 7 .

\section{REFERENCES}

1. R. ENSEY, Isomorphism invariants for Abelian groups modulo bounded groups, Pacific J. Math. 23 (1967).

2. L. Fuchs, Abelian groups, Publishing House of the Hungarian Academy of Sciences, Budapest, 1958.

3. P. Gabriel, Des categories abeliennes, Bull. Soc. Math. France 90 (1962), 323-448.

4. G. Kolettis, Direct sums of countable groups, Duke Math. J. 27 (1960), 111-125.

5. E. A. WALKER, Quotient categories and quasi-isomorphisms of Abelian groups, Proceedings of the colloquim on Abelian groups, ed. by L. Fuchs and E. T. Schmidt, Budapest, 1964, 147-163.

Received January 8, 1968.

TRINITY UNIVERSITY 




\section{Pacific Journal of Mathematics \\ Vol. 29, No. 1 \\ May, 1969}

Jorge Alvarez de Araya, A Radon-Nikodým theorem for vector and operator

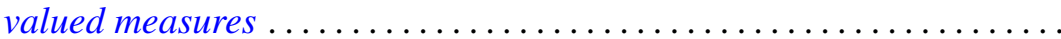

Deane Eugene Arganbright, The power-commutator structure of finite

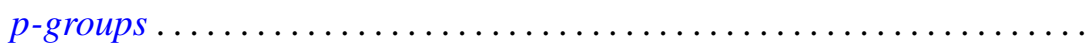

Richard Eugene Barlow, Albert W. Marshall and Frank Proschan, Some

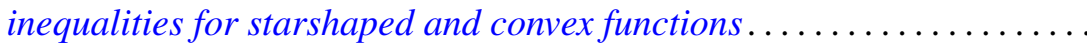

David Clarence Barnes, Some isoperimetric inequalities for the eigenvalues

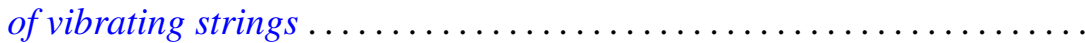

David Hilding Carlson, Critical points on rim-compact spaces ...........

Allan Matlock Weber Carstens, The lattice of pretopologies on an arbitrary

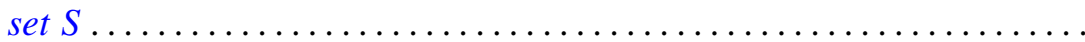

S. K. Chatterjea, A bilateral generating function for the ultraspherical

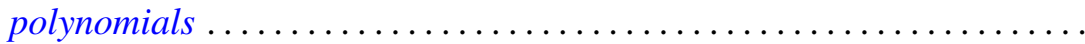

Ronald J. Ensey, Primary Abelian groups modulo finite groups ......... 77

Harley M. Flanders, Relations on minimal hypersurfaces ............ 83

Allen Roy Freedman, On asymptotic density in n-dimensions........... 95

Kent Ralph Fuller, On indecomposable injectives over artinian rings...... 115

George Isaac Glauberman, Normalizers of p-subgroups in finite groups . . . 137

William James Heinzer, On Krull overrings of an affine ring ........... 145

John McCormick Irwin and Takashi Ito, A quasi-decomposable abelian group without proper isomorphic quotient groups and proper isomorphic subgroups.

Allan Morton Krall, Boundary value problems with interior point boundary conditions

John S. Lowndes, Triple series equations involving Laguerre

polynomials

Philip Olin, Indefinability in the arithmetic isolic integers

Ki-Choul Oum, Bounds for the number of deficient values of entire functions whose zeros have angular densities..

R. D. Schafer, Standard algebras ....................

Wolfgang M. Schmidt, Irregularities of distribution. III.

Richard Alfred Tapia, An application of a Newton-like method to the Euler-Lagrange equation 